\title{
Elementos facilitadores ou dificultadores do processo de Transição do Cuidado: uma revisão integrativa
}

\author{
Facilitating or hindering elements of the Care Transition process: na integrative review \\ Elementos que facilitan o dificultan el proceso de Transición del Cuidado: una revisión integradora
}

Recebido: 20/08/2021 | Revisado: 29/08/2021 | Aceito: 03/09/2021 | Publicado: 05/09/2021

\author{
Danieli Bandeira \\ ORCID: https://orcid.org/0000-0003-3700-0728 \\ Universidade Federal de Santa Maria, Brasil \\ E-mail: danielibandeira22@gmail.com \\ Teresinha Heck Weiller \\ ORCID: https://orcid.org/0000-0003-2531-0155 \\ Universidade Federal de Santa Maria, Brasil \\ E-mail: weiller2@hotmail.com \\ Adalvane Nobres Damaceno \\ ORCID: https://orcid.org/0000-0002-4681-0602 \\ Universidade do Vale do Rio dos Sinos, Brasil \\ E-mail: adalvane.damaceno@gmail.com \\ Vanessa Rodrigues Pucci \\ ORCID: https://orcid.org/0000-0002-4036-316X \\ Universidade Federal de Santa Maria, Brasil \\ E-mail: vanessarpucci@hotmail.com \\ Elisa Rucks Megier \\ ORCID: https://orcid.org/0000-0003-3448-9193 \\ Universidade Federal de Santa Maria, Brasil \\ E-mail: elisa.rucks@gmail.com \\ Geraldo de Freitas de Souza Junior \\ ORCID: https://orcid.org/0000-0002-1378-3193 \\ Universidade Federal de Santa Maria, Brasil \\ E-mail: gefreitasjr@gmail.com
}

\begin{abstract}
Resumo
Objetivo: sumarizar na literatura os elementos que facilitam ou dificultam a transição do cuidado de usuários adultos na perspectiva do enfermeiro e/ou usuários de serviços de internação hospitalar cirúrgica para o domicílio ou Atenção Primária à Saúde. Materiais e método: trata-se de uma revisão integrativa da literatura. A busca aconteceu no mês de janeiro de 2021, nas bases de dados Literatura Latino-Americana e do Caribe em Ciências da Saúde, National Library of Medicine/ National Institutes of Health e SciVerse Scopus. Para a seleção dos estudos, utilizou-se a classificação de níveis de evidência. Resultados: Foram localizadas 731 produções, destas, 23 compuseram o corpus do estudo. Os elementos identificados na literatura como facilitadores ou dificultadores do processo de transição do cuidado foram: condução do planejamento de alta, acompanhamento ambulatorial, monitoramento e gerenciamento de sintomas após a alta, educação e promoção do autogerenciamento, segurança das medicações, organização, clareza, disponibilidade, em tempo hábil de informações, comunicação e coordenação do cuidado entre membros da equipe de saúde. Conclusões: estudos como este, são fundamentais para dar visibilidade ao enfermeiro na transição do cuidado, e refletir sobre a importância do trabalho articulado em redes nos diferentes níveis de atenção à saúde.

Palavras-chave: Cuidado transicional; Enfermagem; Atenção primária à saúde; Continuidade da assistência ao paciente; Assistência integral à saúde.

Abstract

Objective: to summarize in the literature the elements that facilitate or hinder the transition of care from adult users from the perspective of nurses and/or users of surgical hospitalization services to the home or Primary Health Care. Materials and method: this is a integrative literature review. The search took place in January 2021, in the Latin American and Caribbean Literature in Health Sciences, National Library of Medicine/National Institutes of Health and SciVerse Scopus databases. For the selection of studies, the classification of levels of evidence was used. Results: A total of 731 productions were located, of which 23 made up the corpus of the study. The elements identified in the literature as facilitating or hindering the care transition process were: conducting discharge planning, outpatient follow-up, monitoring and managing symptoms after discharge, education and promotion of self-management, medication safety, organization, clarity, availability, timely information, communication and coordination of care
\end{abstract}


between members of the health team. Conclusions: studies like this one are essential to give visibility to nurses in the transition of care, and to reflect on the importance of articulated work in networks at different levels of health care.

Keywords: Transitional care; Nursing; Primary helth care; Continuity of patient care; Comprehensive health care.

\section{Resumen}

Objetivo: resumir en la literatura los elementos que facilitan o dificultan la transición de la atención de usuarios adultos desde la perspectiva de enfermeros y / o usuarios de servicios de hospitalización quirúrgica hacia el hogar o Atención Primaria de Salud. Materiales y método: esta es una literatura integradora revisión. La búsqueda se realizó en enero de 2021, en las bases de datos de Literatura Latinoamericana y del Caribe en Ciencias de la Salud, Biblioteca Nacional de Medicina / Institutos Nacionales de Salud y SciVerse Scopus. Para la selección de estudios se utilizó la clasificación de niveles de evidencia. Resultados: Se localizaron un total de 731 producciones, de las cuales 23 conformaron el corpus del estudio. Los elementos identificados en la literatura como facilitadores o entorpecedores de la transición del proceso de atención fueron: realización de la planificación del alta, seguimiento ambulatorio, monitoreo y manejo de síntomas después del alta, educación y promoción del autocuidado, seguridad de la medicación, organización, claridad, disponibilidad, información oportuna, comunicación y coordinación de la atención entre los miembros del equipo de salud. Conclusiones: estudios como este son fundamentales para dar visibilidad al enfermero en la transición asistencial y para reflexionar sobre la importancia del trabajo articulado en redes en los diferentes niveles de la atención de la salud.

Palabras clave: Cuidado de transición; Enfermería; Atención primaria de salud; Continuidad de la atención al paciente; Atención integral de salud.

\section{Introdução}

A transição do cuidado compreende um conjunto de ações com a finalidade de assegurar a coordenação e continuidade dos cuidados de saúde na transferência de usuários entre os diversos serviços de saúde ou entre unidades de um mesmo serviço. Entende-se como o intervalo de tempo entre o início da preparação do usuário para alta até o momento que o mesmo é recebido no próximo serviço (Coleman \& Boult, 2003).

Essas ações contribuem para a redução de internações hospitalares e reinternações causadas por complicações (Gunadi et al., 2015). Entretanto, quando realizada de maneira inadequada pode suscitar eventos adversos, omissão e duplicação de cuidados, atrasos ou recebimentos inadequados nos tratamentos, aumento da mortalidade, insatisfação tanto para familiares quanto profissionais, e sobretudo uso inapropriado dos serviços de saúde e incrementos nos custos (World Health Organization [WHO] 2016).

Desse modo, o preparo envolve planejamento de alta e as ações de educação em saúde com o usuário e familiares, organização logística, articulação entre os serviços de saúde e comunicação entre os profissionais envolvidos no processo colaboram para a minimização de intercorrências relacionadas ao processo de transição (Burke, Kripalani, Vasilevskis \& Schnipper, 2013). Logo, a transição do cuidado mostra-se como potente dispositivo no auxílio da superação da fragmentação da atenção e garantia da continuidade dos cuidados na Rede de Atenção à Saúde (RAS), sendo uma importante estratégia para a efetivação de um sistema integrado de saúde (Acosta, Lima, Marques, Levandovski \& Weber, 2017).

A transição do cuidado do hospital para o domicílio, necessita de alguns domínios voltados para a atenção integral. Esses aspectos, considerados componentes-chave para uma transição ideal, incluem dez domínios. O primeiro deles é o planejamento da alta, considerado uma importante ação para garantir uma transição do hospital para o domicílio segura e eficiente. Outro domínio, a coordenação do cuidado entre membros da equipe de saúde, contempla a sincronização dos cuidados entre os profissionais da saúde nos diferentes serviços de saúde; comunicação de intercorrências, que se refere ao conteúdo do resumo de alta do usuário ou mecanismos de transferência de informação entre a RAS com os cuidados pós-alta. A organização, clareza, disponibilidade, em tempo hábil de informações, considerada um domínio fundamental, deve ser de pronto acesso aos demais serviços de saúde e de fácil entendimento das informações que foram fornecidas antes de assumir o cuidado do usuário (Burke et al., 2013). 
O domínio de segurança das medicações visa informar sobre os medicamentos e auxiliar para a não ocorrência dos eventos adversos pós-alta. A educação e promoção do autogerenciamento, corresponde a educação de usuários e seus familiares, ou cuidadores sobre os principais diagnósticos e instruções do hospital visando o autocuidado, além disso incluem orientações sobre alterações de medicamentos, consultas e com quem entrar em contato se surgirem problemas. $\mathrm{O}$ monitoramento e gerenciamento de sintomas após a alta, conceituados como o tratamento precoce de novos sintomas, agravantes, efeitos colaterais de medicamentos, discrepâncias ou não adesão e outros desafios de autogerenciamento; acompanhamento ambulatorial com profissionais adequados após a alta hospitalar que tem por finalidade garantir transições ideais com a continuidade da assistência ao usuário (Burke et al., 2013).

O planejamento antecipado dos cuidados, envolve as ações que se iniciam no ambiente hospitalar ou ambulatorial e se referem ao estabelecimento e registro de objetivos e preferências dos usuários sobre os cuidados que estão dispostos a receber, considerado fundamental nos cuidados paliativos. Por fim, o suporte psicossocial é um domínio que tem se destacado na composição de uma transição do cuidado ideal, através de programas que oferecem auxílio aos usuários com as atividades domésticas, a alimentação e outras necessidades durante a recuperação, muito úteis para o período de transição (Burke et al., 2013).

Efetivas transições do cuidado reduzem as chances de readmissões hospitalares. Nos Estados Unidos da América (EUA) após incentivos privados e parcerias para melhorar a qualidade da transição do cuidado entre os anos de 2007 a 2012, houve redução das taxas de readmissões dentro de 30 dias após a alta hospitalar (Gerhard et al, 2013). Estudo brasileiro realizado em serviço de emergência, identificou que 33\% dos usuários com doenças crônicas foram readmitidos após 90 dias da alta (Acosta, 2016).

As evidências indicam que efetivas transições são um desafio nos serviços de saúde, em especial aquelas realizadas com usuários cirúrgicos. Entre os momentos que fazem parte da transição do cuidado, são considerados importantes o preparo de alta, as orientações e a abordagem do enfermeiro, pois garantem a retomada das atividades de usuários ao seu domicílio, ainda que esses possam receber alta hospitalar em uso de dispositivos invasivos (drenos, colostomias e outros) e curativos. Posto este cenário, pode ser considerado um problema para o usuário e seus familiares, devido a tensão, estresse e falta de conhecimentos dos cuidados a serem implementados neste contexto (Martins et al, 2015).

Diante o exposto, o presente artigo tem como objetivo sumarizar na literatura os elementos que facilitam ou dificultam a transição do cuidado de usuários adultos na perspectiva do enfermeiro e na perspectiva dos usuários de serviços de internação hospitalar cirúrgica para o domicílio ou Atenção Primária à Saúde (APS).

\section{Metodologia}

Trata-se de uma revisão integrativa de literatura que teve como questão norteadora: quais os elementos que facilitam ou dificultam a transição do cuidado de usuários adultos na perspectiva do enfermeiro e na perspectiva dos usuários de serviços de internação hospitalar cirúrgica para o domicílio ou Atenção Primária à Saúde (APS)? A questão norteadora foi construída pela estratégia PICo, na qual o acrônimo "P" é descrito como população (usuários adultos e/ou enfermeiros), o "I" é descrito como interesse (elementos que facilitam ou dificultam a transição do cuidado) e o "Co" corresponde ao contexto (serviço de internação cirúrgica do hospital para o domicílio ou APS).

A busca foi realizada no mês de janeiro de 2021, utilizando as bases de dados Literatura Latino-Americana e do Caribe em Ciências da Saúde (LILACS), National Library of Medicine/ National Institutes of Health (PubMed) e SciVerse Scopus (SCOPUS), de forma que as estratégias foram adaptadas de acordo com as características de cada base de dados. Para o desenvolvimento das estratégias de busca foi realizado um mapeamento dos termos o qual envolveu os descritores, sinônimos e palavras relacionadas à pergunta de revisão. Os descritores foram identificados segundo o índice dos Descritores em Ciência 
da Saúde (DeCs) da Biblioteca Virtual de Saúde (BVS), e Medical Subject Headings (MeSH). Após a definição e combinação dos descritores foram utilizados os operadores boleanos “OR”, “AND” e "NOT” para se obter as estratégias utilizadas (quadro 1). Na base de dados PUBMED, obteve-se 329 publicações, na LILACS, se obteve 262 publicações e na SCOPUS, obteve-se 140 publicações.

Quadro 1. Estratégias de busca nas bases de dados PubMed, LILACS e SCOPUS.

\begin{tabular}{|c|c|}
\hline Base & Estratégia \\
\hline PubMed & 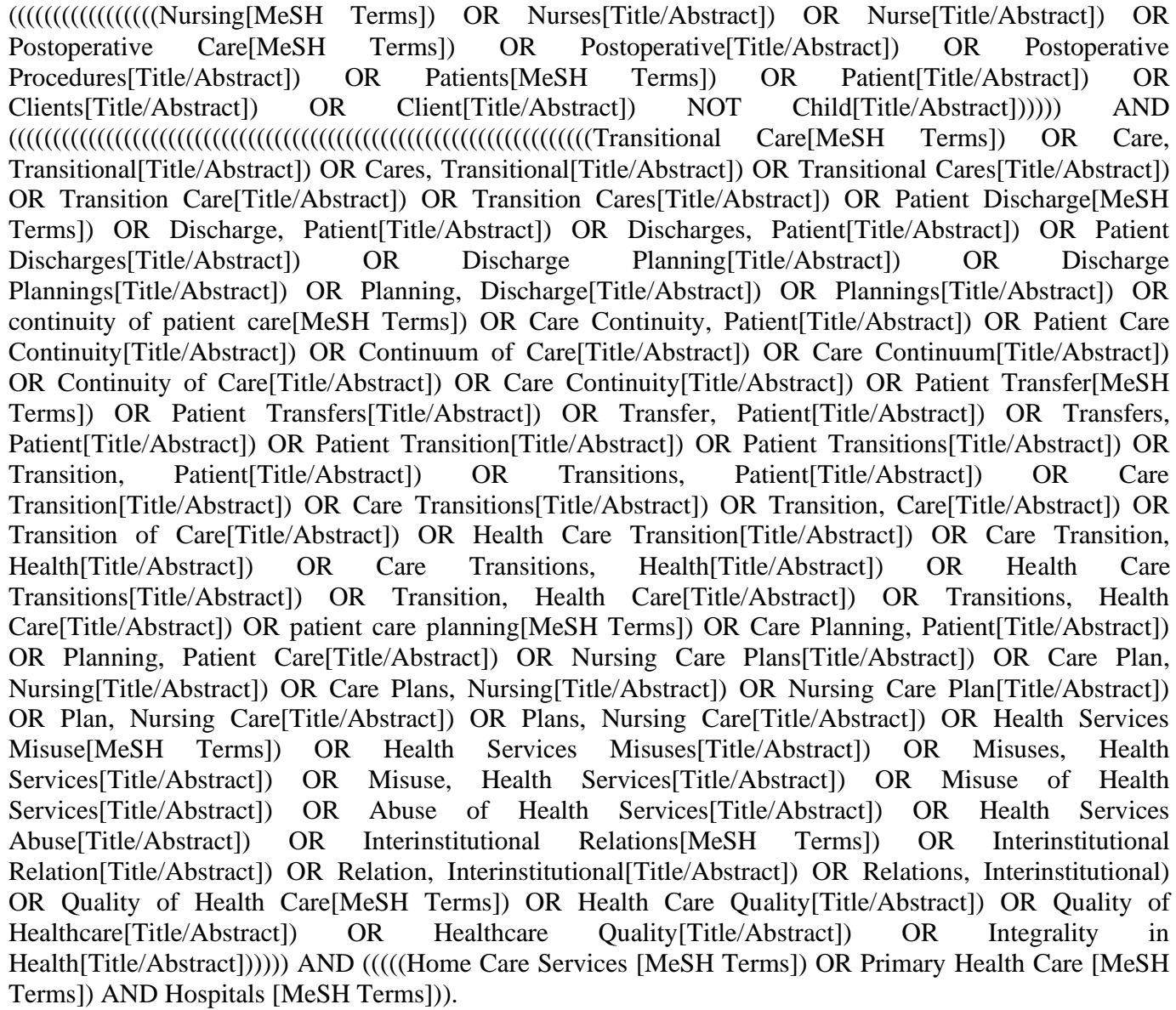 \\
\hline $\mathrm{ACS}$ & $\begin{array}{l}\text { (tw:((tw:(enfermagem)) OR (tw:(enfermeiros)) OR (tw:(enfermeira)) OR (tw:(enfermeiras)) OR } \\
\text { (tw:(enfermeiro)) OR (tw:(paciente)) OR (tw:(pacientes)) OR (tw:(cliente)) OR (tw:(clientes)) OR } \\
\text { (tw:(cuidados pós-operatórios)) OR (tw:(Assistência no período pós-operatório)) OR (tw:(Assistência na } \\
\text { fase pós-operatória)) OR (tw:(Assistência pós-operatória )) OR (tw:(Cuidados pós-cirúrgicos)) AND NOT } \\
\text { (tw:(criança)))) AND (tw:((tw:(Cuidado transicional)) OR (tw:(Cuidado de Transição)) OR (tw:(Cuidados } \\
\text { de Transição)) OR (tw:(Tratamento Transicional)) OR (tw:(Tratamento de Transição)) OR (tw:(Alta do } \\
\text { paciente) OR (tw:(Planejamento da Alta)) OR (tw:(Alta do Hospital)) OR (tw:(Alta Hospitalar)) OR } \\
\text { (tw:(Continuidade da assistência ao paciente)) OR (tw:(Acompanhamento dos Cuidados de Saúde)) OR } \\
\text { (tw:(Transferência da Responsabilidade pelo Paciente)) OR (tw:(Transferência do Paciente)) OR } \\
\text { (tw:(Planejamento de Assistência ao Paciente)) OR (tw:(Plano de Tratamento)) OR (tw:(Plano de Cuidados } \\
\text { de Enfermagem)) OR (tw:(Mau Uso de Serviços de Saúde )) OR (tw:(Má Prestação de Serviços de Saúde)) } \\
\text { OR (tw:(Relações Interinstitucionais )) OR (tw:(Relações entre Organizações)) OR (tw:(Qualidade da } \\
\text { Assistência à Saúde)) OR (tw:(Qualidade dos Cuidados de Saúde)) OR (tw:(Qualidade dos Serviços de } \\
\text { Saúde)) OR (tw:(Qualidade Assistencial)) OR (tw:(Integralidade em Saúde)) OR (tw:(Globalidade dos } \\
\text { Cuidados)) OR (tw:(Globalidade em Saúde Integralidade)))) AND (tw:(tw:((tw:(serviços de assistência } \\
\text { domiciliar)) OR (tw:(cuidado domiciliar)) OR (tw:(serviços de cuidados domiciliares)) OR (tw:(serviços } \\
\text { residenciais terapêuticos)) OR (tw:(atenção primária à saúde)) OR (tw:(atençãa primária de saúde)) OR } \\
\text { (tw:(atenção básica)) OR (tw:(atenção básica à saúde)) OR (tw:(atenção básica de saúde) OR (tw:(cuidados } \\
\text { de saúde primários)) AND (tw:(cirurgia geral)) OR (tw:(cirurgia)) AND (tw:(hospital)) OR (tw:(centro } \\
\text { hospitalar)) OR (instance:"regional")) AND (instance:"regional"))). }\end{array}$ \\
\hline
\end{tabular}




\begin{tabular}{|l|l|}
\hline \multirow{2}{*}{ SCOPUS } & ((TITLE-ABS-KEY ("Transitional Care") OR TITLE-ABS-KEY ("health care transitions") OR TITLE- \\
ABS-KEY ("Transitional Cares") OR TITLE-ABS-KEY ("Transition Care") OR TITLE-ABS-KEY \\
("Patient Discharge") OR TITLE-ABS-KEY ("Discharge Planning") OR TITLE-ABS-KEY ("continuity of \\
patient care") OR TITLE-ABS-KEY ("Patient Care Continuity") OR TITLE-ABS-KEY ("Continuum of \\
Care") OR TITLE-ABS-KEY ("Patient Transfer") OR TITLE-ABS-KEY ("Patient Transition") OR TITLE- \\
ABS-KEY ("Care Transition") OR TITLE-ABS-KEY ("patient care planning") OR TITLE-ABS-KEY \\
("Nursing Care Plans") OR TITLE-ABS-KEY ("Health Services Misuse") OR TITLE-ABS-KEY \\
("Interinstitutional Relations") OR TITLE-ABS-KEY ("Quality of Health Care") OR TITLE-ABS-KEY \\
("Quality of Healthcare"))) AND ((TITLE-ABS-KEY (nursing) OR TITLE-ABS-KEY (nurses) OR TITLE- \\
ABS-KEY ("postoperative care") OR TITLE-ABS-KEY (patient) OR TITLE-ABS-KEY (clients) AND \\
NOT TITLE-ABS-KEY (child))) AND ((TITLE-ABS-KEY ("home care services") OR TITLE-ABS-KEY \\
("primary health care") AND TITLE-ABS-KEY (hospitals) AND TITLE-ABS-KEY (surgery) OR TITLE- \\
ABS-KEY ("postoperativ period"))).
\end{tabular}

Fonte: Autores (2021).

Em relação aos critérios de elegibilidade, foram incluídos artigos originais publicados na íntegra, disponíveis em inglês, espanhol ou português, independente da abordagem metodológica. Não foi adotado recorte temporal.

Dentre os elementos que foram considerados para a análise da transição do cuidado foram considerados alguns domínios, são eles: planejamento da alta, comunicação de intercorrências, organização, clareza e disponibilidade, em tempo hábil de informações, segurança das medicações, educação do usuário e promoção do autogerenciamento, suporte psicossocial, planejamento antecipado dos cuidados, coordenação do cuidado entre membros da equipe de saúde, monitoramento e gerenciamento de sintomas após a alta e acompanhamento ambulatorial (Burke et al., 2013).

A seleção das produções foi realizada por meio do duplo independente, a fim de evitar possíveis vieses. Primeiramente foi realizada a leitura de títulos e resumos e selecionados os estudos que respondessem à questão de revisão. Posteriormente, foi realizada a leitura das produções na íntegra. Para auxiliar na seleção dos estudos, foi utilizado o software Mendeley® e realizado o preenchimento de uma ficha de registro de exclusão das produções contendo o número de identificação do artigo e os motivos da exclusão: não é artigo; não tem no idioma; não é pesquisa; não é da temática; não responde à questão de pesquisa.

Para a revisão crítica dos estudos, utilizou-se a classificação de níveis de evidência (Melnyk \& Fineout-Overhol, 2011). A classificação em níveis de evidência foi construída considerando o tipo de questão clínica do estudo primário. Conforme a questão clínica há três pirâmides de classificação de evidências, sendo a primeira relacionada a questões tratamento ou intervenção na área da saúde, podendo ser classificada em sete níveis de evidência, a segunda envolve prognóstico ou etiologia na área da saúde, composta por cinco níveis de evidência e a terceira quanto ao significado, experiência ou compreensão dos sentimentos, também com cinco níveis de evidência. Ressalta-se que quanto menor o número do nível, maior é a sua força de evidência.

Após a análise das produções incluídas foi organizada e preenchida uma ficha de extração documental, composta pelos seguintes itens: identificação do artigo, ano de publicação, título, autor, procedência, objetivo, método, nível de evidência, principais resultados e elementos da transição do cuidado na perspectiva do enfermeiro e/ou usuário.

\section{Resultados}

Foram localizadas 731 produções, destas, 23 compuseram o corpus do estudo (Figura 1). Os títulos e resumos foram lidos concomitantemente, sendo excluídos aqueles que não respondiam à questão da pesquisa ou estivessem duplicados. 
Figura 1 - Fluxograma da coleta de dados e seleção dos estudos que compuseram a amostra.

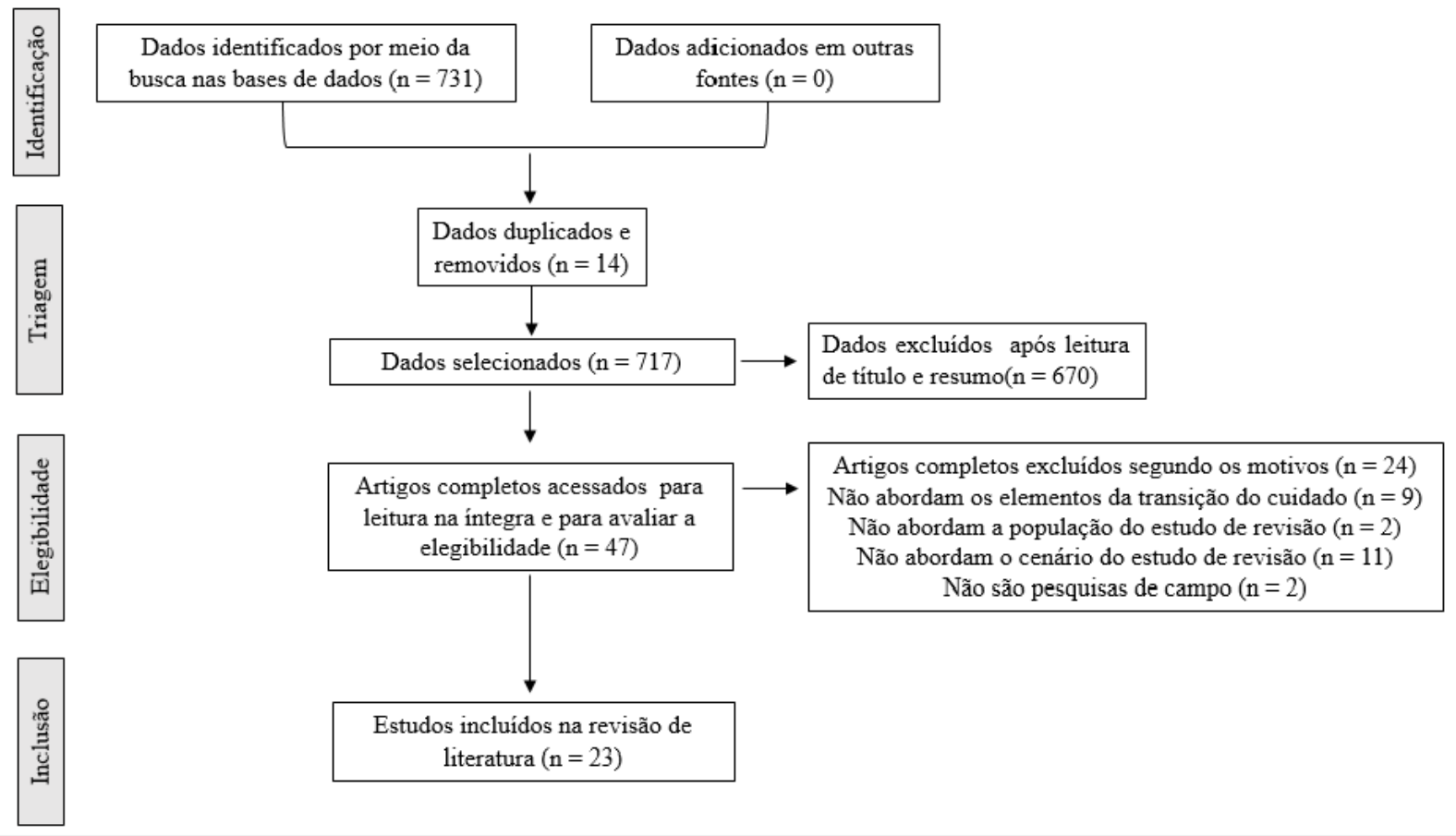

Fonte: Autores (2021).

Quanto ao país de procedência dos estudos houve predominância de artigos publicados nos EUA, contabilizando oito produções (34,78\%), seguidos do Canadá, com quatro produções (17,39\%), Espanha com três produções (13,04\%), Reino Unido com duas produções $(8,7 \%)$, Brasil, Holanda, Noruega, Colômbia, Turquia e Austrália contabilizaram uma produção cada $(4,35 \%)$.

Quanto à distribuição temporal, a maior parte dos estudos 56,52\% (n=13) foram publicados entre os anos de $2015 \mathrm{e}$ 2019. Os anos de 2010 a 2014 correspondem a 17,39\% das produções (n=4), bem como os anos 2005 a 2009 que também representam $17,39 \%$ produções $(n=4)$, os anos 2000 a 2004 contabilizam 8,7\% das produções $(n=2)$.

Quanto ao nível de evidência, a maior parte das pesquisas (60,87\%) está classificada no nível 2. A análise dos estudos possibilitou a identificação dos elementos facilitadores e dificultadores da transição do cuidado de usuários de serviços de internação hospitalar cirúrgica para o domicílio/APS (Quadro 2).

Quadro 2 - Quadro sinóptico das produções incluídas sobre os elementos que facilitam e dificultam a transição do cuidado de usuários de serviços de internação hospitalar cirúrgica para o domicílio/APS.

\begin{tabular}{|c|c|c|c|c|}
\hline Artigo & Objetivo & $\begin{array}{l}\text { Abordagem } \\
\text { metodológica }\end{array}$ & Elementos da transição do cuidado & NE \\
\hline $\begin{array}{l}\text { Rebollo } \\
\text { et al. } \\
(2017)\end{array}$ & $\begin{array}{l}\text { Criar uma rota de atendimento de cirurgia ambulatorial } \\
\text { de grande porte (CMA) em que uma maior colaboração } \\
\text { com a Atenção Básica (AB) no início e continuidade } \\
\text { do processo permita simplificar a atividade pré- } \\
\text { operatória e a programação cirúrgica, além de otimizar } \\
\text { o acompanhamento até o final do episódio clínico. }\end{array}$ & Quantitativo & $\begin{array}{l}\text { Facilitador: Coordenação entre os níveis de atenção; } \\
\text { Planejamento de alta por meio de protocolos, orientações } \\
\text { e guias. }\end{array}$ & 6 \\
\hline
\end{tabular}




\begin{tabular}{|c|c|c|c|c|}
\hline $\begin{array}{l}\text { Cunha et } \\
\text { al. } \\
(2016)\end{array}$ & $\begin{array}{l}\text { Compreender os fatores que influenciam o processo de } \\
\text { referência e contrarreferência da pessoa com } \\
\text { indicação/submetida à Cirurgia de Revascularização do } \\
\text { Miocárdio no cenário da Atenção Primária à Saúde. }\end{array}$ & Qualitativo & $\begin{array}{l}\text { Facilitador: APS no fortalecimento do vínvulo, acesso a } \\
\text { consultas e exames, e retirada de medicamentos pela } \\
\text { APS; Ações gerenciais do enfermeiro na APS. } \\
\text { Dificultador: Deficit de orientações e acolhimento na } \\
\text { APS; Falta de profissionais da APS e necessidade de } \\
\text { aprimoramento dos profissionais; Fila de espera para } \\
\text { consultas médicas na APS e transferências para outros } \\
\text { níveis; Falhas de comunicação; Entraves na gestão } \\
\text { municipal; Sistema informatizado desintegrado entre os } \\
\text { níveis de atenção. }\end{array}$ & 2 \\
\hline $\begin{array}{l}\text { Travieso } \\
\text { e Ruiz, } \\
(2009)\end{array}$ & $\begin{array}{l}\text { Avaliar o papel da Enfermagem de } \mathrm{AB} \text { na } \\
\text { implementação, desenvolvimento e controle de } \\
\text { qualidade do processo hipertrofia benigna da próstata - } \\
\text { câncer de próstata. }\end{array}$ & Quantitativo & $\begin{array}{l}\text { Facilitador: cuidado de enfermagem na implementação e } \\
\text { execução do processo, guiando uma diminuição } \\
\text { significativa no atraso na referência. }\end{array}$ & 2 \\
\hline $\begin{array}{l}\text { Brooke, } \\
\text { Beckstro } \\
\text { m, } \\
\text { Slager, } \\
\text { Weir e } \\
\text { Del Fiol } \\
(2019)\end{array}$ & $\begin{array}{l}\text { Caracterizar até que ponto os fatores associados à } \\
\text { recuperação do paciente mais velho, como status } \\
\text { funcional, status cognitivo, status social e fatores } \\
\text { emocionais, são compartilhados entre os PCPs e os } \\
\text { prestadores de cirurgia durante a transição do } \\
\text { atendimento. }\end{array}$ & Qualitativo & $\begin{array}{l}\text { Dificultador: intercâmbio de informações - pouca troca } \\
\text { de informações entre o PCP e o cirurgião; gerenciar } \\
\text { medicamentos; cuidados fragmentado. }\end{array}$ & 2 \\
\hline $\begin{array}{l}\text { Aued, } \\
\text { Bernardi } \\
\text { no, } \\
\text { Lapierre } \\
\text { e } \\
\text { Dallaire } \\
(2019)\end{array}$ & $\begin{array}{l}\text { Descrever as atividades desenvolvidas pelas } \\
\text { enfermeiras de ligação para a continuidade dos } \\
\text { cuidados após a alta hospitalar. }\end{array}$ & Qualitativo & $\begin{array}{l}\text { Facilitador: A transferência de informações é reforçada } \\
\text { pela entrega de alguns documentos aos usuários com } \\
\text { alta, que podem ser entregues aos profissionais de } \\
\text { cuidados primários ou a outros serviços. }\end{array}$ & 2 \\
\hline $\begin{array}{l}\text { Kind et } \\
\text { al. } \\
(2012)\end{array}$ & $\begin{array}{l}\text { Avaliar o Programa de Cuidados de Transição } \\
\text { Coordenada (C-TraC) que foi projetado para melhorar } \\
\text { a coordenação de cuidados e os resultados entre os } \\
\text { veteranos com condições de alto risco descarregados } \\
\text { para ambientes comunitários do William S. Middleton } \\
\text { Memorial Veterans Hospital, em Madison, Wisconsin. }\end{array}$ & Quantitativo & $\begin{array}{l}\text { Facilitador: protocolo; reconciliação de medicamento e } \\
\text { aconselhamento de alta no hospital. Contato via telefone } \\
\text { dentro de } 48-72 \mathrm{~h} \text { após a alta para reforçar os objetivos } \\
\text { do cuidado de transição. } \\
\text { Dificultador: discrepância de medicação identificada e } \\
\text { corrigida; enfermeiro responsável pelo caso não estava } \\
\text { disponível nos finais de semana e feriados, não } \\
\text { implementando o protocolo. }\end{array}$ & 4 \\
\hline $\begin{array}{l}\text { Langelaa } \\
\mathrm{n}, \\
\text { Baines, } \\
\text { Bruijne } \\
\mathrm{e} \\
\text { Wagner } \\
(2017)\end{array}$ & $\begin{array}{l}\text { Avaliar a presença,exatidão e oportunidade das cartas } \\
\text { de alta hospitalar registros. }\end{array}$ & Quantitativo & $\begin{array}{l}\text { Dificultador: Cartas de alta que estão disponíveis muitas } \\
\text { vezes faltam componentes. }\end{array}$ & 4 \\
\hline $\begin{array}{l}\text { Horstma } \\
\mathrm{n} \text { et al. } \\
(2017)\end{array}$ & $\begin{array}{l}\text { Examinar o papel das instruções de alta na pós- } \\
\text { cuidados de alta para pacientes submetidos a } \\
\text { tratamento com cirurgia colorretal e relatar temas } \\
\text { relacionados à percepção do paciente de instruções de } \\
\text { alta e experiência pós-alta. }\end{array}$ & Qualitativo & $\begin{array}{l}\text { Facilitador: instruções de alta proporcionaram } \\
\text { segurança, removeram dúvidas, lembrete verbal e } \\
\text { educação fornecida no hospital. } \\
\text { Dificultador: instruções fornecidas foram insuficientes, } \\
\text { experiências negativas após a alta quando os prestadores } \\
\text { de cuidados não apreciaram a importância de seus } \\
\text { sintomas ou não reconheceram preocupação expressa } \\
\text { que exigia intervenção oportuna. }\end{array}$ & 2 \\
\hline $\begin{array}{l}\text { Meredith } \\
\mathrm{e} \\
\text { Emberto } \\
\mathrm{n}(2000)\end{array}$ & $\begin{array}{l}\text { Examinar a escala atual de fornecimento de materiais } \\
\text { de informação ao paciente por cirurgiões consultores } \\
\text { no NHS do Reino Unido e em hospitais do setor } \\
\text { privado. }\end{array}$ & Quantitativo & $\begin{array}{l}\text { Dificultador: ausência de provisão rotineira de materiais } \\
\text { de informação de suporte aos pacientes submetidos à } \\
\text { cirurgia. }\end{array}$ & 4 \\
\hline $\begin{array}{l}\text { Van } \\
\text { Walrave } \\
\mathrm{n} \text { et al. } \\
(2008)\end{array}$ & $\begin{array}{l}\text { Determinar a probabilidade de que as informações do } \\
\text { paciente de visitas anteriores com outros médicos } \\
\text { estava disponível para uma visita médica atual. }\end{array}$ & Quantitativo & $\begin{array}{l}\text { Facilitador: informações da visita anterior, disponível na } \\
\text { visita atual. } \\
\text { Dificultador: o tipo de médico influenciou na } \\
\text { disponibilidade de informações, sendo maior quando o } \\
\text { médico atual era médico da família. }\end{array}$ & 2 \\
\hline
\end{tabular}




\begin{tabular}{|c|c|c|c|c|}
\hline $\begin{array}{l}\text { Hardima } \\
\mathrm{n}, \\
\text { Reames, } \\
\text { McLeod } \\
\text { e } \\
\text { Regenbo } \\
\text { gen } \\
(2016)\end{array}$ & $\begin{array}{l}\text { Projetar e implementar uma lista de verificação } \\
\text { centrada no paciente para promover independência e } \\
\text { validação do conhecimento de autocuidado e } \\
\text { habilidades de cuidado, e avaliar seu efeito sobre } \\
\text { readmissões após a criação da ileostomia. }\end{array}$ & Quantitativo & $\begin{array}{l}\text { Facilitador: implementação de uma educação pós- } \\
\text { operatória centrada no paciente e orientada para o } \\
\text { autocuidado foi associada a chances reduzidas de } \\
\text { readmissão após a criação de ileostomia em cirurgia } \\
\text { colorretal. }\end{array}$ & 2 \\
\hline $\begin{array}{l}\text { Wahlber } \\
\mathrm{g}, \\
\text { Braaten } \\
\mathrm{e} \\
\text { Broderst } \\
\text { ad } \\
(2016)\end{array}$ & $\begin{array}{l}\text { Avaliar o efeito da implementação do modelo de } \\
\text { referência para quatro grupos de diagnóstico - } \\
\text { dispepsia, suspeita de câncer colorretal, tóraxdor e } \\
\text { pulmonar obstrutiva crônicadoença (DPOC) - no } \\
\text { encaminhamento do paciente. }\end{array}$ & Quantitativo & $\begin{array}{l}\text { Facilitador: Interação e informação. } \\
\text { Dificultador: falta de informação ao especialista. }\end{array}$ & 2 \\
\hline $\begin{array}{l}\text { Carrillo } \\
\text { et al. } \\
(2019)\end{array}$ & $\begin{array}{l}\text { Avaliar o efeito de uma intervenção educacional } \\
\text { (CUIDAR) em pacientes com câncer durante o período } \\
\text { pós operatório, que visa fortalecer a competência para } \\
\text { cuidar do paciente em casa nas dimensões } \\
\text { conhecimento, unicidade instrumental, gozo, } \\
\text { antecipação e relações e interações sociais. }\end{array}$ & Quantitativo & $\begin{array}{l}\text { Facilitador: intervenção educativa; aconselhamento } \\
\text { telefônico; formar um grupo de profissionais que podem } \\
\text { fornecer apoio permanente, dando instruções curtas e } \\
\text { simples, orientações sobre a transição do hospital para } \\
\text { casa e uso da rede de suporte disponível. }\end{array}$ & 4 \\
\hline $\begin{array}{l}\text { Keller, } \\
\text { Merchan } \\
\text { t, } \\
\text { Commo } \\
\text { n e } \\
\text { Laizner } \\
(2017)\end{array}$ & $\begin{array}{l}\text { Examinar as experiências dos pacientes de alta } \\
\text { hospitalar e preparação para encaminhamento para } \\
\text { serviços de atendimento domiciliar de } \\
\text { acompanhamento. }\end{array}$ & Qualitativo & $\begin{array}{l}\text { Facilitardor: preparação para o encaminhamento } \\
\text { relacionada a informação e orientações. } \\
\text { Dificultador: enfermeiro de cuidados domiciliares } \\
\text { despreparado; erro na transferência de informações. }\end{array}$ & 2 \\
\hline $\begin{array}{l}\text { Dash e } \\
\text { Pichkeri } \\
\text { ng } \\
(2017)\end{array}$ & $\begin{array}{l}\text { Avaliar e melhorar a qualidade da comunicação da } \\
\text { Atenção Básica à Saúde na alta com foco nos cuidados } \\
\text { pós-operatórios. }\end{array}$ & Quantitativo & $\begin{array}{l}\text { Facilitador: cartão de informações de alta do paciente. } \\
\text { Dificultador: ausência de informações relevantes sobre a } \\
\text { ferida cirúrgica, técnicas de fechamento utilizadas e } \\
\text { gestão contínua. }\end{array}$ & 4 \\
\hline $\begin{array}{l}\text { Acher et } \\
\text { al. } \\
(2015)\end{array}$ & $\begin{array}{l}\text { Avaliar como as transições de cuidados se relacionam } \\
\text { e influenciam a readmissão da perspectiva do paciente } \\
\text { e do médico usando a Systems Engineering Initia-para } \\
\text { o modelo de Segurança do Paciente (SEIPS). }\end{array}$ & Misto & $\begin{array}{l}\text { Dificultador: pouca compreensão do paciente e cuidador; } \\
\text { preparação de alta inadequada para o atendimento } \\
\text { domiciliar; processo educacional e } \text { materiais } \\
\text { insuficientes; comunicação inadequada da equipe } \\
\text { assistencial; déficits na coordenação da equipe } \\
\text { assistencial e na gestão de medicação. }\end{array}$ & 4 \\
\hline $\begin{array}{l}\text { Hall et } \\
\text { al. } \\
(2014)\end{array}$ & $\begin{array}{l}\text { Projetar e testar um inovador programa de cuidados de } \\
\text { transição, envolvendo cirurgia cardíaca profissionais } \\
\text { de enfermagem. }\end{array}$ & Quantitativo & $\begin{array}{l}\text { Facilitador: programa de transição para casa que oferece } \\
\text { continuidade do atendimento, centro de comunicação e } \\
\text { gestão da medicação por meio de profissionais de } \\
\text { enfermagem do hospital reduziu significativamente a } \\
\text { readmissão em } 30 \text { dias e morte após CABG. }\end{array}$ & 2 \\
\hline $\begin{array}{l}\text { Brooke } \\
\text { et al. } \\
(2014)\end{array}$ & $\begin{array}{l}\text { Testar se o acompanhamento do Provedor de Cuidados } \\
\text { Primários (PCP) está associado a taxas mais baixas de } \\
\text { readmissão em } 30 \text { dias após reparo aberto de } \\
\text { aneurisma de aorta torácica (TAA) e reparo de hérnia } \\
\text { ventral (VHR). }\end{array}$ & Quantitativo & $\begin{array}{l}\text { Facilitador: o acompanhamento de rotina com um PCP } \\
\text { após a cirurgia de alto risco é um mecanismo eficaz e de } \\
\text { baixo custo para limitar readmissões. }\end{array}$ & 2 \\
\hline $\begin{array}{l}\text { Dal, } \\
\text { Bulut e } \\
\text { Demir } \\
(2012)\end{array}$ & $\begin{array}{l}\text { Determinar os problemas vivenciados por pacientes } \\
\text { operados em casa no primeiro mês após a cirurgia. }\end{array}$ & Quantitativo & $\begin{array}{l}\text { Dificultador: Controle da dor; edema/circulação; } \\
\text { exercícios e atividades físicas e autocuidado em casa. } \\
\text { Necessidade de planejamento individualizado, apoiado } \\
\text { por ferramentas aduditivas; fornecimentos de } \\
\text { treinamento de alta, aconselhamento por meio de } \\
\text { telefene, serviço de atendimento domiciliar. }\end{array}$ & 4 \\
\hline $\begin{array}{l}\text { Herrera } \\
\text { et al. } \\
(2011)\end{array}$ & $\begin{array}{l}\text { Determinar o impacto, sobre o uso de serviços de } \\
\text { saúde e ansiedade do paciente, de um programa de } \\
\text { assistência por telefone durante o mês seguinte à alta } \\
\text { de uma cirurgia de trauma, juntamente com análise de } \\
\text { orçamento. }\end{array}$ & Quantitativo & $\begin{array}{l}\text { Facilitador: } O \text { atendimento por telefone reduz as visitas } \\
\text { para a sala de emergência. } \\
\text { Dificultador: o regime terapêutico foi a principal causa } \\
\text { de dúvidas dos pacientes após a alta cirúrgica do trauma. }\end{array}$ & 2 \\
\hline
\end{tabular}




\begin{tabular}{|l|l|l|l|l|}
\hline $\begin{array}{l}\text { Morin et } \\
\text { al. } \\
(2009)\end{array}$ & $\begin{array}{l}\text { Avaliar o processo de implementação de uma modelo } \\
\text { de gerenciamento de cuidados de enfermagem } \\
\text { comunitário e avaliar os efeitos desse modelo em } \\
\text { pacientes acompanhados em casa. }\end{array}$ & $\begin{array}{l}\text { Quali- } \\
\text { quantitativo }\end{array}$ & $\begin{array}{l}\text { Facilitador: o modelo de gestão de cuidados de } \\
\text { enfermagem foi considerado útil o suficiente para ser } \\
\text { integrado na prática de cuidados de rotina em lares de } \\
\text { idosos após cirurgia cardíaca. }\end{array}$ & $\begin{array}{l}\text { Dificultador: necessidades de pacientes e cuidadores por } \\
\text { informações e apoio após a cirurgia de CRM. }\end{array}$ \\
\hline $\begin{array}{l}\text { Theobal } \\
\text { McMurr } \\
\text { ay } \\
(2004)\end{array}$ & $\begin{array}{l}\text { Examinar a gama de problemas pós-alta, preocupações } \\
\text { e necessidades dos pacientes e seus cuidadores } \\
\text { fo miliares após a alta após cirurgia de revascularização } \\
\text { necessidades não atendidas um ano depois. }\end{array}$ & Qualitativo \\
\hline
\end{tabular}

Fonte: Dados da pesquisa, Santa Maria/RS (2021).

\section{Discussão}

Para a discussão dos resultados, optou-se pela abordagem individualizada de cada domínio da transição do cuidado, suas facilidades ou dificuldades, com o intuito de possibilitar a apreensão detalhada dos elementos da transição.

No tocante ao domínio "Planejamento de Alta" os estudos indicaram a criação de protocolos de alta em cooperação com o hospital e APS, com orientações e guias para os serviços (Rebollo et al., 2017; Kind et al., 2012; Acher et al., 2015). Ressalta-se a importância de programas ou protocolos de transição para casa, liderados por enfermeiros, a fim de prestar um aconselhamento de alta e garantir a continuidade do atendimento, comunicação, gestão da medicação, reduzindo as taxas de readmissão hospitalar (Kind et al., 2012; Hall et al., 2014).

Os estudos direcionam que para que o planejamento de alta seja eficaz, é necessário que o processo de educação e informações sejam fornecidas ao usuário face a face desde o ingresso na instituição, além de workshops e acompanhamento telefônico durante um mês após a alta hospitalar (Carrillo et al., 2019). Para isso, é necessário que nas equipes de saúde, haja a formação de um grupo de profissionais que possam fornecer feedback e apoio permanente, dando instruções curtas e simples orientações sobre a transição do hospital para casa e o uso das RAS disponíveis (Carrillo et al., 2019).

O planejamento de alta antecipado está atrelado a redução significativa das readmissões hospitalares e o tempo de permanência dessas readmissões. Há uma série de fatores durante a transição do cuidado que podem contribuir para a readmissão, por exemplo, a compreensão do usuário e cuidador, a preparação de alta inadequada para o atendimento domiciliar, a falha no processo educacional (Acher et al., 2015).

O domínio "Coordenação do cuidado entre membros da equipe de saúde" é essencial para garantir a continuidade dos cuidados e o uso adequado dos recursos em saúde (Rebollo et al., 2017). A coordenação da atenção entre os níveis da RAS, no cenário brasileiro, é atributo ordenador da APS para garantia do acesso universal e igualitário às ações e serviços de saúde (Portaria $\mathrm{n}^{\circ}$ 2.436, 2017). Os estudos trazem a utilização da APS para retirada de medicamentos, acesso a consultas e exames como fatores que facilitam o fortalecimento do vínculo entre os usuários e APS, sendo este um fator favorável no processo de transição na RAS (Cunha et al., 2016).

Entretanto, a falta de profissionais e necessidade de aprimoramento destes, as filas de espera que prejudicam o acesso dos usuários ao serviço, a demora em referenciar o usuário para especialistas na média complexidade para definição do seu diagnóstico quando necessário e entraves de questões de gestão municipal que acabam por impedir que o processo de referência se concretize são os dificultadores do processo no âmbito da APS (Cunha et al., 2016).

A escassez de troca de informações entre a APS e a equipe de cirurgia também foi citada como uma aliada na fragmentação do cuidado. Pesquisa evidenciou que a maioria dos usuários (67\%), sentem-se os principais responsáveis por comunicar seu próprio estado de saúde a APS e a equipe de cirurgia, incluindo informações sobre status funcional, status social e status cognitivo (Brooke et al., 2019). Corroborando com outro estudo que destaca os erros de organização na transferência 
de informações entre as equipes de saúde, destacando que a RAS atua de maneira desintegrada e desarticulada (Keller et al., 2017).

Neste sentido, o fortalecimento da RAS, torna-se fundamental, uma vez que o acompanhamento de rotina com a APS, após cirurgia de alto risco foi associado a uma menor probabilidade de readmissão em 30 dias. Logo, é importante que seja assegurado ao usuário o direito de acompanhamento dos cuidados primários, pois trata-se de um mecanismo eficaz e de baixo custo para eliminar readmissões (Brooke et al., 2014; Morin et al., 2009)

No que se refere a "Comunicação de intercorrências", estudos apontam fragilidades no acompanhamento do usuário pela APS, devido a falhas na comunicação entre a APS e os serviços de alta complexidade após a realização da cirurgia. Soma- se que o principal responsável por informar sua situação de saúde, o que foi realizado no serviço especializado, suas condições clínicas, a ocorrência do episódio cirúrgico, é o próprio usuário (Cunha et al., 2016; Brooke et al., 2019). Ressalta-se que esta comunicação, por vezes inadequada, interfere diretamente na qualidade das transições do cuidado, e contribui para a readmissão hospitalar (Acher et al., 2015).

A “Organização, clareza, disponibilidade, em tempo hábil de informações” perpassa pela adequada comunicação entre os profissionais da RAS, como principal estratégia de comunicação, encontra-se o prontuário eletrônico, que contém informações sobre o usuário, as quais podem ser compartilhadas entre a APS e média complexidade. Entretanto a alta complexidade utiliza um sistema informatizado desintegrado do sistema utilizado na APS, interrompendo o fluxo de informações e fragilizando o processo de contrarreferência (Cunha et al., 2016).

Assim, a não utilização de um sumário de alta ou carta de alta, inviabiliza o processo de transição do cuidado, repercutindo diretamente na assistência ao usuário (Cunha et al., 2016; Langelaan et al., 2017). Estudo traz que em 70\% das readmissões não planejadas, a carta de alta do índice de admissão estava faltando ou estava incompleta no momento da readmissão (Horstman et al., 2017). Outro estudo revela que o tipo de hospital interfere na probabilidade de disponibilidade de informação, sendo maior para usuários internados em hospitais acadêmicos. A especialidade médica também influenciou a disponibilidade de informações, sendo maior quando era médico da família em vez de um especialista (Van Walraven et al., 2008).

Destaca-se o papel fundamental do cuidado de enfermagem, na implantação e execução do processo, guiando uma diminuição significativa no atraso da referência e transferência de usuários para cuidados especializados, ajuste adequado aos critérios de qualidade do processo nas referências feitas (Travieso \& Ruiz, 2009). A transferência de informações do usuário é reforçada pela entrega de alguns documentos a eles mesmos, como prescrições de alta, resumo de alta, folhetos informativos, cartão de informações de alta, entre outros, que podem ser entregues aos profissionais de cuidados primários ou a outros serviços para que saibam o que aconteceu durante a internação hospitalar e como eles podem continuar o tratamento do usuário (Aued et al., 2019; Keller et al., 2017; Dash \& Pichkering, 2017).

Essas informações são fundamentais para que seja implantado um cuidado integral, porém, o que se observa em alguns estudos é que nem sempre as informações são fornecidas aos demais serviços para garantir a continuidade da assistência (Wahlberg et al., 2016). Para que as informações sejam levantadas em tempo hábil, é importante que se faça melhorias nos materiais educacionais e que seja aumentada a coordenação da equipe assistencial, com um melhor engajamento e compreensão do usuário e cuidador. Isso tudo é essencial para otimizar as transições do cuidado visando a redução da readmissão, bem como, melhorar a saúde no "pós-alta" (Acher et al., 2015).

Quanto ao domínio "Segurança das medicações" evidencia-se que a reconciliação medicamentosa na alta hospitalar é um fator crítico na transição dos cuidados pós-hospitalares (Lee et al., 2017; Brooke et al., 2019; Kind et al., 2012; Hall et al., 2014). Estudo com 849 usuários adultos submetidos a cirurgia cardíaca eletiva, apontou lacunas na reconciliação dos 
medicamentos prescritos, o que acarreta em interrupções inadequadas que variam de $18,8 \%$ a 52,3\% para medicamentos individuais (Lee et al., 2017).

Em estudo que avaliou a implantação de um protocolo de transição do cuidado, foi observado discrepância de medicação em quase metade dos participantes, no período de dois ou três dias após a alta hospitalar, ainda que todos usuários tivessem reconciliação medicamentosa e aconselhamento para alta no hospital (Kind et al., 2012). Ressaltando a importância dos ensinamentos de reforço após a alta, de maneira otimizada a fim de auxiliar na administração dos regimes de medicação em domicílio.

A "Educação e Promoção do autogerenciamento" através das instruções de alta proporcionam uma sensação de segurança aos usuários, os mesmos reconhecem o papel das instruções de alta como um lembrete verbal de educação, capacitando-os para assumir o controle de seus cuidados (Horstman et al., 2017). Contudo, os estudos indicam que a maioria dos usuários consideram as instruções a eles passadas, insuficientes para ter acesso eficaz ao médico oportuno e eficaz nos cuidados pós alta (Horstman et al., 2017). Este déficit de informações no momento da alta hospitalar é percebido por usuários dos serviços públicos e privados. Um estudo evidenciou que usuários tratados em hospitais privados eram menos propensos a receber materiais informativos em comparação com os tratados em serviços públicos, indicando que a disponibilidade de informações escritas é desigual e varia de acordo com a política do profissional e instituição que o atendeu (Meredith \& Emberton, 2000).

Neste sentido, evidencia-se a importância da implementação de uma "educação pós-operatória" centrada no usuário e orientada para o autocuidado, uma vez que, se compreende que a educação é uma estratégia viável que aumenta a capacidade de cuidar e reduz significativamente as readmissões após procedimento cirúrgico (Hardiman et al., 2016; Carrillo et al., 2019; Acher et al., 2015). É importante salientar que a educação do usuário perpassa pela capacitação do seu autogerenciamento, sendo condição importante que os prestadores de cuidados de saúde compartilhem informações, não apenas relacionadas ao suporte profissional, mas também orientações sobre o acompanhamento do cuidado domiciliar (Keller et al., 2017).

A educação de usuários cirúrgicos, perpassa informações relevantes de cuidado com a ferida cirúrgica, técnicas de fechamento utilizadas e sua avaliação contínua. Em estudo que avaliou práticas de cuidados de primários, identificou que são de baixo conhecimento (Dash \& Pichkering, 2017). Outro ponto a ser destacado, que interfere diretamente na educação e autogerenciamento dos usuários, é o déficit de orientações e acolhimento do usuário na APS (Cunha et al., 2016).

Já o domínio "Monitoramento e gerenciamento de sintomas após a alta" destaca o importante papel da APS por meio de ações gerenciais utilizadas por enfermeiros e a equipe de saúde. Esses profissionais são responsáveis por favorecer o acesso à APS, a criação de vínculo entre equipe de saúde e o usuário. Além disso, são facilitadores do processo de identificação precoce de sinais e sintomas das comorbidades de base do usuário e referência na RAS (Cunha et al., 2016).

Considera-se uma estratégia promissora de monitoramento e gerenciamento de sintomas os telefonemas realizados por parte da equipe hospitalar, por intermédio de um enfermeiro de ligação, em horário pré-combinado dentro de 48 a 72 horas após a alta, a fim de reforçar os objetivos de cuidado de transição. Essas ligações têm continuidade semanal até que a equipe ou usuários não veja mais necessidade (Kind et al., 2012; Carrillo et al., 2019; Dal, Bulut \& Demir, 2012). Estudo experimental que realizou consultas telefônicas para acompanhamento de usuários pós-cirúrgicos, revelou um aumento de custos de 4.149,32 euros. Contudo, este grupo gerou 25 visitas a menos nos serviços de urgência do que o grupo controle, implicando uma economia de 3.649,25 euros. A diferença de 500 euros equivale a um custo médio de 1,65 euros para cada um dos 302 participantes incluídos no programa (Herrera et al., 2011).

O domínio “Acompanhamento ambulatorial” revela-se importante, haja vista, as necessidades tanto dos usuários como dos cuidadores, por informações e apoio após cirurgia, com acompanhamento dos profissionais após a alta, evidenciando falhas no acompanhamento ambulatorial (Theobald \& McMurray, 2004). 
Outro estudo com usuários pós-cirurgia cardíaca, relata que estes são orientados a continuar o acompanhamento de saúde no ambulatório da instituição hospitalar onde realizou a cirurgia. Isso torna-se importante para dar continuidade ao acompanhamento do usuário após a cirurgia, considerando-se aspectos como reabilitação cardíaca e necessidade de mudanças no estilo de vida, diante da falta de estrutura e preparo dos profissionais da APS para acompanhar este usuário (Cunha et al., 2016).

Ainda sobre a falta de estrutura da APS, o número reduzido de Agentes Comunitários de Saúde (ACS) nas equipes de Estratégia de Saúde da Família (ESF) é evidenciado como um entrave no processo de contrarreferência, repercutindo de forma negativa no acompanhamento após a cirurgia destes usuários, uma vez que a necessidade de visitas domiciliares está comumente atrelada a estes. Dessa maneira, por vezes, a APS não toma conhecimento do usuário que realizou a cirurgia, pois o elo entre a APS com a comunidade fica comprometido (Cunha et al., 2016).

Os estudos em questão não trouxeram contribuições acerca dos domínios "Planejamento antecipado dos cuidados" e "Suporte psicossocial", evidenciando lacunas nas ações dos profissionais da saúde durante a transição do cuidado com usuários cirúrgicos. Sabe-se que quando um domínio não é abordado ou é pouco desenvolvido, a transição do cuidado pode ser deficiente quando o usuário chega ao ambiente domiciliar (Burke et al., 2013). Logo, a ausência de um ou mais domínios na transição do cuidado, nos convida a refletir sobre este processo, as reais necessidades dos usuários e a capacidade de articulação e coordenação da RAS, a fim de garantir uma atenção integral e de qualidade.

Definiu-se para esta revisão os domínios da transição do cuidado propostos por Burke e colaboradores (2013), ou seja, os elementos facilitadores ou dificultadores da transição do cuidado foram analisados e estruturados conforme estes domínios, e configurando este aspecto como uma limitação do estudo. Além disso, o estudo incluiu em sua população usuários adultos e/ou enfermeiros, o que não permitiu generalizar os elementos para outras populações, incluindo a pediátrica ou demais profissionais da saúde.

\section{Considerações Finais}

A revisão possibilitou a identificação dos elementos que facilitam ou dificultam a transição do cuidado de usuários de serviços de internação hospitalar cirúrgico para o domić́lio. Os elementos da transição do cuidado identificados nos estudos envolveram uma série de componentes estratégicos, são eles: planejamento de alta, acompanhamento ambulatorial, monitoramento e gerenciamento de sintomas após a alta, educação e promoção do autogerenciamento, segurança das medicações, organização, clareza, disponibilidade, em tempo hábil de informações, comunicação e coordenação do cuidado entre membros da equipe de saúde.

Essas estratégias foram realizadas pela equipe multiprofissional, em especial, pelo enfermeiro, o qual assumiu destaque nas ações evidenciadas pelos estudos. Elementos como o planejamento antecipado dos cuidados, e suporte psicossocial não figuraram entre as estratégias de transição de usuários cirúrgicos, o que indica uma lacuna nos domínios que compõem o processo de transição do cuidado. Ademais, nenhum estudo apresentou nível de evidência 1, de modo que há uma lacuna no que diz respeito à prática baseada em evidências.

Observa-se que o tema em questão ainda é incipiente no Brasil, principalmente no tocante a transição de usuários cirúrgicos. Estudos como este são fundamentais para dar visibilidade ao papel do enfermeiro na transição do cuidado, além disso, nos convida a refletir sobre a importância do trabalho articulado em redes nos diferentes níveis de atenção à saúde, apontando a necessidade do fortalecimento da política das Redes de Atenção à Saúde (RAS) e criação e implementação de protocolos de transição do cuidado voltados aos serviços de internação cirúrgica.

Diante disso, considera-se importante o desenvolvimento de novas revisões que permitam ampliar a abrangência de estudos primários, bem como a população do estudo, incluindo outros profissionais de saúde e usuários pediátricos, por 
exemplo. Ademais, evidencia-se a necessidade do desenvolvimento de estudos de campo que avaliem a qualidade da transição do cuidado dos serviços de internação cirúrgica para o domicílio ou APS, a fim de aprofundar o conhecimento acerca da temática e suscitar melhorias na articulação da RAS.

\section{Referências}

Coleman, E. A., \& Boult, C. (2003) American Geriatrics Society Health Care Systems Committee. Improving the quality of transitional care for persons with complex care needs. J Am Geriatr Soc, 51(4):556-7.

Gunadi, S., Upfield, S., Pham, N. D., Yea, J., Schmiedeberg, M. B., \& Stahmer, G. D. (2015). Development of a collaborative transitions-of-care program for heart failure patients. Am J Health-Syst Pharm,72(13):1147-52.

World Health Organization. (2016). Transitions of Care: Technical Series on Safer Primary Care. World Health Organization. https://apps.who.int/iris/bitstream/handle/10665/252272/9789241511599-eng.pdf;jsessionid=F02 F4867BC0581E4B21DE72B23FC0994?sequence=1

Burke, R. E., Kripalani, S., Vasilevskis, E. E., \& Schnipper, J. L. (2013). Moving beyond readmission penalties: creating an ideal process to improve transitional care. J Hosp Med, 8(2):102-9.

Acosta, A. M., Lima, M. A. D., Marques, G. Q., Levandovski, P. F., \& Weber, L. A. F. (2017). Brazilian version of the Care Transitions Measure: translation and validation. Int Nurs Rev, 64(3):379-87.

Gerhard, G., Yemane, A., Hickman, P., Oelschlaeger, A., Rollins, E., \& Brennan, N. (2013). Data Shows Reduction in Medicare Hospital Readmission Rates During 2012. Medicare \& Medicaid Research Review, 3(2).

Acosta, A. M. (2016). Transição do cuidado de pacientes com doenças crônicas: do serviço de emergência para o domicílio. Tese de doutorado, Programa de Pós-Graduação em Enferamgem, Universidade Federal do Rio Grande do Sul, Porto Alegre.

Martins, K. P., Costa, K. N., Macedo, K. N. F., Oliveira, D. S. T., Valdevino, S. C., \& Rezende, L. C. (2015). Nurse's role on preparing for discharge of surgical patients. J. res.: fundam. Care, 07(1):1756-1764.

Melnyk, B. M., \& Fineout-Overhol, T. E. (2011). Evidence-based practice in nursing \& healthcare. A guide to best practice. Philadelphia:Lippincot Williams \& Wilkins.

Rebollo, G. A. M., Álvarez, A. I., Fernández, G. C. L., Arondo, K. M. I., Alonso, C. E., \& Colina, A. A. (2017). Integration of primary health care in outpatient surgery programs. Kirubide Project. Cir. mayor ambul, 22(4), 199-201.

Lee, J. S., Gonzales, R., Vittinghoff, E., Corbett, K. K., Fleischmann, K. E., Sehgal, N., \& Auerbach, A. D. (2017). Appropriate Reconciliation of Cardiovascular Medications After Elective Surgery and Postdischarge Acute Hospital and Ambulatory Visits. J Hosp Med, 12(9), 723-730.

Cunha, K. S., Higashi, G. D. C., Erdmann, A. L., Kahl, C., Koerich, C., \& Meirelles, B. H. S. (2016) Myocardial revascularization: factors intervening in the reference and counter-reference in Primary Health Care. TT. Esc. Enferm. USP, 50(6), 965-972.

Travieso, M. J. M. \& Ruiz, A. J. M. (2009). Proceso asistencial hipertrofia benigna de próstata-cáncer de próstata: papel de enfermería de atención primaria en la implantación, desarrollo y control de calidad del processo/No disponible. ENFURO: Rev. Asoc. Esp. A.T.S. Urol, 110, 10-12.

Brooke,. B. S., Beckstrom, J., Slager, S. L., Weir, C. R. \& Del Fiol, G. (2019). Discordance in Information Exchange Between Providers During Care Transitions for Surgical Patients. J Surg Res, 244, 174-180.

Aued, G. K., Bernardino, E., Lapierre, J. \& Dallaire, C. (2019). Liaison nurse activities at hospital discharge: a strategy for continuity of care. Rev. Latino-Am. Enfermagem, 27,3162.

Kind, A. J. H., Jensen, L., Barczi, S., Bridges, A., Kordahl, B., Smith, M. A., \& Asthana, S. (2012). Low-Cost Transitional Care With Nurse Managers Making Mostly Phone Contact With Patients Cut Rehospitalization At A VA Hospital. Health Affairs (Project Hope), 31(12), 2659-2668.

Langelaan, M., Baines, R. J., Bruijne, M. C. \& Wagner, C. (2017). Association of admission and patient characteristics with quality of discharge letters: posthoc analysis of a retrospective study. BMC Health Services Research, 17:225

Horstman, M. J., Mills, W. L., Herman, L. I., Cai, C., Shelton, G., Qdaisat, T., Berger, D. H., \& Naik, A. D. (2017). Patient experience with discharge instructions in postdischarge recovery: a qualitative study. BMJ Open, 7(2), 014842.

Meredith, P. \& Emberton, M. (2000). The NHS patient information lottery: it is whom you see rather than what you need. Annals of The Royal College of Surgeons of England, 82(3), 217-222.

Van Walraven, C., Taljaard, M., Bell, C. M., Etchells, E., Zarnke, K. B., Stiell, I. G., \& Forster, A. J (2008). Information exchange among physicians caring for the same patient in the community. CMAJ: Canadian Medical Association Journal, 179(10), 1013-1018.

Hardiman, K. M., Reames, C. D., McLeod, M. C. \& Regenbogen, S. E. (2016). A Patient-Autonomy-Centered Self-Care Checklist Reduces Hospital Readmissions After Ileostomy Creation. Surgery, 160(5), 1302-1308.

Wahlberg, H., Braaten, T. \& Broderstad, A. R. (2016). Impact of referral templates on patient experience of the referral and care process: A cluster randomised trial. BMJ Open, 6(10). 
Research, Society and Development, v. 10, n. 11, e417101119636, 2021

(CC BY 4.0) | ISSN 2525-3409 | DOI: http://dx.doi.org/10.33448/rsd-v10i11.19636

Carrillo, G., Mesa, M., Gómez, O.J., Numpaque, O. \& Laguna, M. (2019). Educational intervention (CUIDAR) in cancer patients treated with surgery: A quasi-experimental study. Patient Education and Counseling, 102(8), 1475-1482.

Keller, G., Merchant, A., Common, C. \& Laizner, A. M. (2017). Patient experiences of in-hospital preparations for follow-up care at home. Journal of Clinical Nursing, 26(11-12), 1485-1494.

Dash, I. \& Pickering, G. T. (2017). Improving post-operative communication between primary and secondary care: The wound closure information card. Primary Health Care Research and Development, 18(1), 92-96.

Acher, A. W., Lecaire, T. J., Hundt, A. S., Greenberg, C. C., Carayon, P., Kind, A. J., \& Weber, S. M. (2015). Using human factors and systems engineering to evaluate readmission after complex surgery. Journal of the American College of Surgeons, 221(4), 810-820.

Hall, M. H., Esposito, R. A., Pekmezaris, R., Lesser, M., Moravick, D., Jahn, L., Blenderman, R., Akerman, M., Nouryan, C. N., \& Hartman, A. R. (2014) Cardiac surgery nurse practitioner home visits prevent coronary artery bypass graft readmissions. Annals of Thoracic Surgery, 97(5), 1488-1495.

Brooke, B. S, Stone, D. H., Cronenwett, J. L., Nolan, B., De Martino, R. R., MacKenzie, T. A., Goodman, D. C., \& Goodney P. P. (2014). Early primary care provider follow-up and readmission after high-risk surgery. JAMA Surgery, 149(8), 821-828.

Dal, Ü., Bulut, H. \& Demir, S. G. (2012). The problems experienced by the patients at home after surgery. Medical Journal of Bakirkoy, 8(1), 34-40.

Herrera, E. C., Rodríguez, A. M. D. M., Navarro, E. J. L., Godoy, M. A., García, P. A., Rodríguez, J. G., \& Sánchez, I. R. (2011). Effect of a telephone care program after hospital discharge from a trauma surgery unit. Gaceta Sanitaria, 25(2), 133-138.

Morin, D., Aubin, M., Vezina, L., Gagnon, J., Racine, S., Reinharz, D., Paradis, M., Dallaire, C., \& Aubin, K. (2009). From hospital to home after cardiac surgery: Evaluation of a community nursing care management model. Professional Case Management, 14(4), 167-175.

Theobald, K. \& McMurray, A. (2004). Coronary artery bypass graft surgery: Discharge planning for successful recovery. Journal of Advanced Nursing, 47(5), 483-491.

Portaria n 2.436, de 21 de setembro de 2017 (2017). Aprova a Política Nacional de Atenção Básica, estabelecendo a revisão de diretrizes para a organização da Atenção Básica, no âmbito do Sistema Único de Saúde (SUS). https://bvsms.saude.gov.br/bvs/saudelegis/gm/2017/prt2436_22_09_2017.html. 